\title{
BMJ Open Impact of integrated upper limb spasticity management including botulinum toxin A on patient-centred goal attainment: rationale and protocol for an international prospective, longitudinal cohort study (ULIS-III)
}

\begin{abstract}
Lynne Turner-Stokes, ${ }^{1}$ Stephen Ashford,${ }^{1}$ Jorge Jacinto, ${ }^{2}$ Pascal Maisonobe, ${ }^{3}$ Jovita Balcaitiene, ${ }^{3}$ Klemens Fheodoroff ${ }^{4}$
\end{abstract}

To cite: Turner-Stokes $L$, Ashford S, Jacinto J, et al. Impact of integrated upper limb spasticity management including botulinum toxin $\mathrm{A}$ on patient-centred goal attainment: rationale and protocol for an international prospective, longitudinal cohort study (ULIS-III). BMJ Open 2016;6: 011157. doi:10.1136/bmjopen-2016011157

- Prepublication history for this paper is available online. To view these files please visit the journal online (http://dx.doi.org/10.1136/ bmjopen-2016-011157).

Received 14 January 2016 Revised 30 March 2016 Accepted 26 April 2016

CrossMark

For numbered affiliations see end of article.

Correspondence to Professor Lynne TurnerStokes;

lynne.turner-stokes@doctors. org.uk

\section{ABSTRACT}

Objectives: Describe the rationale and protocol for the Upper Limb International Spasticity (ULIS)-III study, which aims to evaluate the impact of integrated spasticity management, involving multiple botulinum toxin A (BoNT-A) injection cycles and concomitant therapies, on patient-centred goal attainment. Outline novel outcome assessment methods for ULIS-III and report initial evaluation data from goal setting in early stages of the study.

Design: Large international longitudinal cohort study of integrated upper limb spasticity management, including BoNT-A.

Participants and setting: ULIS-III is a 2-year study expected to enrol >1000 participants at 58 study centres across 14 countries.

Interventions: The study design is non-interventional and intended to reflect real-life clinical practice. It will describe injection practices and additional treatment strategies, and record clinical decision-making in a serial approach to long-term spasticity management.

Outcome measures: ULIS-III will use a goaldirected approach to selection of targeted standardised measures to capture the diversity of presentation, goals and outcomes. ULIS-III will implement the Upper Limb Spasticity Index, a battery of assessments including a structured approach to goal attainment scaling (Goal Attainment ScalingEvaluation of Outcomes for Upper Limb Spasticity tool), alongside a limited set of standardised measures, chosen according to patients' selected goal areas. Concomitant therapy inputs, patient satisfaction with engagement in goal setting, health economic end points and health-related quality of life data will also be captured.

Results of initial evaluation of goal quality: Recruitment started in January 2015. By June 2015, 58 sites had been identified and initial data collected for 79 patients across 13 sites in 3 countries. Goal setting data were quality-checked and centres rated on the basis of function-related and Specific, Measurable, Achievable, Realistic, Timed (SMART) characteristics
Strengths and limitations of this study

- The Upper Limb International Spasticity (ULIS)-III is the first large international longitudinal cohort study to describe real-life clinical practice and decision-making in spasticity management over a 2-year period of treatment.

- The structured approach to goal attainment scaling linked to targeted standardised measures will improve comparability of outcomes while maintaining a patient-centred approach. This approach may also have broader application for outcome measurement in other areas of healthcare.

- This study introduces new tools to capture therapy interventions and spasticity-related quality of life. While not yet fully validated, they address areas that are poorly described in other studies; ULIS-III will enable further validation.

- Training and proactive feedback on the standard of goals set from an early stage in the study has helped to ensure high-quality goal setting.

- This study represents a diverse sample of practice across four continents; however, the limited number of subjects per centre $(n=20-30)$ could lead to a degree of selection bias.

of goal statements. Overall, 11/13 centres achieved the highest rating $\left(A_{++}\right)$.

Conclusions: ULIS-III will provide valuable information regarding treatment of and outcomes from real-life upper limb spasticity management worldwide.

Trial registration number: NCT02454803; Preresults.

\section{INTRODUCTION}

Upper limb spasticity is a common and disabling feature of neurological conditions that affect the central nervous system. ${ }^{1-3}$ It may induce pain, interfere with function or self- 
care and result in secondary complications such as abnormal posture, contracture, pressure areas and resultant deformity. ${ }^{1}$ Regardless of aetiology, active management is required to prevent these unwanted effects. ${ }^{1}$

There is an ever-growing body of evidence that localised injections of botulinum toxin A (BoNT-A) can be effective in the management of upper limb spasticity. ${ }^{4}$ However, the diversity of individual goals for treatment poses a challenge for the evaluation of outcomes from treatment. ${ }^{156}$ Previous studies have identified the principal goal areas of spasticity management, which include improving active and passive function, reducing pain and deformity, avoiding involuntary movements, improving mobility, and facilitating therapies such as postural management, exercise, stretching and strengthening of limbs, and splinting. ${ }^{7}$

Patient-centred goal setting is a fundamental guiding principle of rehabilitation. ${ }^{8-10}$ However, communication between clinicians and patients/caregivers to agree on treatment goals and systematic documentation of goal attainment are still not everyday practice for some rehabilitation teams. ${ }^{11} 12$

Described originally in the 1960 s by Kiresuk and Sherman, ${ }^{13}$ goal attainment scaling (GAS) was introduced in the context of upper limb spasticity management in $2006^{14}$ and has gained recognition over the past decade as an effective method for capturing and quantifying patient-centred goal achievement. ${ }^{6}{ }^{15}$ However, it does not provide directly comparable data; therefore, standard outcome measures must be collected alongside GAS to support comparison between different practices and populations. ${ }^{16}$ Some authors have also called for more standardised goals or 'item banks' to reduce the variation in goal setting. ${ }^{17}$ Owing to this goal diversity, the challenge is to incorporate standardised measures that capture the diverse aims of treatment into a timeeffective approach that is practical for real-life clinical practice.

The Upper Limb International Spasticity (ULIS)-III study will aim to describe real-life clinical practice and decision-making in integrated spasticity management over a 2-year period, using a structured approach to GAS that incorporates targeted standardised measures while maintaining a patient-centred approach.

\section{THE ULIS PROGRAMIME AND THE EVOLUTION OF OUTCOME MEASUREMENT}

The ULIS programme consists of large international observational studies investigating the use of BoNT-A injections as part of an integrated spasticity management strategy for the treatment of upper limb spasticity. The overall aim of this programme is to describe real-life clinical practice and to develop a common approach to outcome measurement that could build into a core data set for ongoing longitudinal evaluation, taking into account the diversity of presentation and clinical approaches to treatment.
The initial stage of the programme, ULIS-I, ${ }^{11}$ was a cross-sectional survey conducted across 122 centres in 31 countries that documented the clinical profiles and treatment goals and reported outcome evaluation in adults receiving BoNT-A treatment for upper limb spasticity. The majority of clinicians $(78 \%)$ reported setting goals, but formal GAS was used in only $5 \%$ of centres. ${ }^{11}$ For the next stage of the programme, Turner-Stokes et $a l^{18}$ concluded that goal attainment may provide an acceptable common outcome measure for this context, if it was applied consistently.

ULIS-II was an international observational study to investigate goal attainment as a primary outcome following a single BoNT-A injection cycle. It was conducted in 84 centres across 22 countries in the European Union, Pacific Asia, Eastern Europe and South America. A key feature of ULIS-II was its development of a consistent standard of goal setting and application of GAS in participating centres, using the GAS-light method. ${ }^{16}$ The approach was then used to quantify and characterise patient-centred goals and to confirm the validity of GAS as an efficient measure of patient outcome. ${ }^{18}$ The study results showed that $80 \%$ of patients $(n=363)$ achieved their primary goals and that the use of an electronic case report form (eCRF) was a feasible approach to data collection for the ULIS study programme. ${ }^{5}$ The study also confirmed the validity of the GAS T-score as an overall assimilation of primary and secondary goal attainment. Clinicians were encouraged to continue to use the standardised measures that they normally applied in routine clinical practice. Although these were diverse, some relationships emerged between the use of specific measures and certain goals for treatment. For example, visual analogue scales were commonly used alongside GAS to record the reduction of pain symptoms or improved ease of care.

ULIS-II formed the basis for development of a more structured approach, framing goals around recommended goal parameters for each goal area and using a limited set of standardised measures from which the subsets used were individually determined according to priority goal areas. These refinements to the GAS-light approach were developed in a proof-of-principle study before progression to the third stage of the ULIS programme. They are described in more detail below.

\section{Rationale for ULIS-III}

Spasticity typically varies over time, both in its severity and in the pattern of muscles affected. Its course may fluctuate, or follow a trajectory of recovery or progression, depending on the underlying condition, its phase of development and treatment. ${ }^{1}$ Ongoing treatment may include further injections of BoNT-A into the same or different muscles, physical interventions (eg, splinting, task practice or exercise, functional electrical stimulation), or the use of other antispasmodic medications. ${ }^{1}$ Longitudinal data are therefore needed to record 
progress over time and to capture clinical decisionmaking along the care pathway.

ULIS-II provided an overview of how treatment goals and outcome measures were applied in a single cycle of treatment with BoNT-A. However, it did not provide insight into the changing course of treatment over time. It recorded concomitant treatments in terms of the number of therapy sessions, but did not provide information on their content. Nor did it attempt to capture information on quality of life or health utility in relation to spasticity management.

The present study, ULIS-III, will expand the patient cohort to evaluate integrated spasticity management that includes multiple BoNT-A treatment cycles and concomitant therapies, as delivered in real-life clinical practice. It will implement a novel approach to outcome measurement using patient-centred goal setting and GAS alongside standardised validated outcome measures, in a generalisable sample, recorded longitudinally over a 2-year period.

\section{NOVEL ASSESSMENT METHODS}

ULIS-III will use the Upper Limb Spasticity Index (ULS Index) to record patient-centred goals and standardised outcomes directed by priority goal areas. This is a battery of assessments designed to capture the diversity of presentation, goals and outcomes inherent in populations of patients with upper limb spasticity, while targeting measurement on the priority areas of treatment for that individual. While the goal-directed approach to selection of targeted standardised measures is novel in this context, the measures themselves are wellvalidated tools and are suitable for use in routine practice.

ULIS-III will also employ some new tools including:

- The Upper Limb Focal Spasticity Therapy Recording Schedule (ULSTR), which records the number, duration and types of therapies related to specific goals;
- The Spasticity-Related Quality-of-Life Tool (SQoL-6D), which records health utility data and is designed specifically to assess aspects of quality of life that are likely to be affected by spasticity.

Although these new instruments have not been fully validated, they address areas that have been poorly documented in previous studies and for which currently available tools are deficient.

Figure 1 summarises the process for application of measures in ULIS-III.

\section{The ULS Index}

The ULS Index is a battery of assessments that can be used to evaluate both patient-rated and clinician-rated components of outcome measurement in upper limb spasticity.

The three main components of the ULS Index are as follows (details provided in table 1):

1. Severity of presentation and confounders to recovery, including:

- Demographics;

- Distribution and severity of spasticity and soft tissue contractures;

- Severity of impairment

- Local to upper limb (eg, motor control, sensory loss, neglect),

- General (eg, cognitive, behavioural, communicative, mood).

2. Individual GAS using a structured approach, including:

- Goal Attainment Scaling-Evaluation of Outcome for Upper Limb Spasticity (GAS-eous): Specific, Measurable, Achievable, Realistic and Timed (SMART) goal setting supported by targeted standard measures according to goal areas;

- Patient satisfaction with and engagement in goal setting.
Figure 1 Process of application of measures in the ULIS-III study. BoNT-A, botulinum toxin A; GAS-eous, Goal Attainment Scaling-Evaluation of Outcome for Upper Limb Spasticity; ULIS-III, third study in the Upper Limb International Spasticity programme.

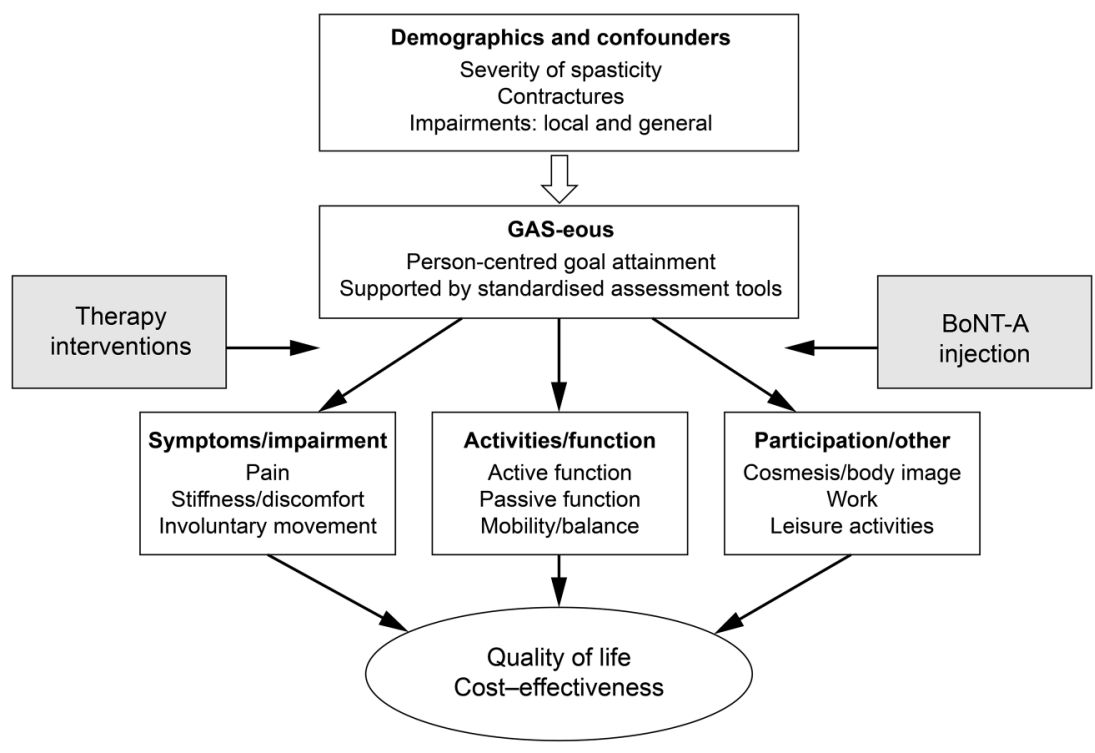


Table 1 Components of the ULS Index

\begin{tabular}{|c|c|c|}
\hline ULS Index & Rated by clinician & Rated by patient/carer \\
\hline \multirow{2}{*}{$\begin{array}{l}\text { A. Severity and confounders (history and } \\
\text { examination) }\end{array}$} & Demographics & NA \\
\hline & $\begin{array}{l}\text { Distribution of spasticity } \\
\text { Severity of spasticity (MAS) } \\
\text { Severity of impairment (ULS-NIS) } \\
\text { Soft tissue contractures (loss of } \\
\text { range) }\end{array}$ & NA \\
\hline \multirow[t]{2}{*}{ B. Goals for treatment } & \multicolumn{2}{|c|}{$\begin{array}{l}\text { GAS-eous tool: SMART goals negotiated between patient and team with } \\
\text { associated measurement parameters within each chosen goal area }\end{array}$} \\
\hline & $\begin{array}{l}\text { Patient engagement in goal } \\
\text { setting }^{21}\end{array}$ & Patient satisfaction with goals ${ }^{21}$ \\
\hline \multicolumn{3}{|c|}{$\begin{array}{l}\text { C. Standardised measures-applied according } \\
\text { to the CHOSEN GOAL AREAS for treatment } \\
\text { Symptoms }\end{array}$} \\
\hline Involuntary movements & $\mathrm{ARRS}^{23}$ & NA \\
\hline Function: passive and active & NA & $\begin{array}{l}\text { ArmA: passive and active } \\
\text { function }^{24} \\
\text { Ease of caring for limb (NGRS) }\end{array}$ \\
\hline Mobility & $\begin{array}{l}\mathrm{FAC}^{25} \\
10 \mathrm{~m} \text { walk (speed) }\end{array}$ & NA \\
\hline Global benefits & Global benefit scale $(-2$ to +2$)$ & Global benefit scale $(-2$ to +2$)$ \\
\hline
\end{tabular}

3. A limited set of standardised measures

- The measures applied are determined by the goal areas, so a standard measure is rated ONLY when that category is one of the goals for treatment.

\section{Standardised measures in the ULS Index}

Standardised outcome measures were selected on the basis that they were supported by published evidence of validity and were already used in clinical practice by centres in ULIS-II.

- The Modified Ashworth Scale (MAS), which reports variations in muscle tone during flexion and extension, measures severity of spasticity. ${ }^{19}$ MAS was selected over other spasticity measures as it was demonstrated in the ULIS-I and ULIS-II studies to be the most widely used tool in routine clinical practice. $^{5} 18$

- The Upper Limb Spasticity-adapted version of the Neurological Impairment Scale (ULS-NIS) measures severity of functional impairment, ${ }^{20}$ and was used in ULIS-II.

- The Numbered Graphic Rating Scale (NGRS) and Scale of Pain Intensity (SPIN) are visual analogue and verbal rating scales for evaluating symptoms including pain, ease of care, ease/difficulty in performing a task and sleep quality, among others. ${ }^{22}$

- The Associated Reaction Rating Scale (ARRS) is used to measure involuntary movements, ${ }^{23}$ for example, those associated with walking.
- The Arm Activity (ArmA) measure is a standardised scale for determining active and/or passive function. ${ }^{24}$

- The Functional Ambulation Category (FAC) ${ }^{25}$ and the $10 \mathrm{~m}$ walk test record walking ability.

\section{The GAS-eous tool}

The GAS-eous tool was designed to provide a structured framework for the application of GAS alongside standardised measures in the context of upper limb spasticity. ${ }^{26}$ Its development was based on a previous secondary analysis $^{7}$ that evaluated goals set for the treatment of upper limb spasticity ( $\mathrm{n}=696$ primary or secondary goals) according to GAS across four single-centre and multicentre studies. ${ }^{6142728}$ GAS for these studies was applied as described by Turner-Stokes, ${ }^{16}$ an adaptation of the method published by Kiresuk and Sherman. ${ }^{13}$ Goal definitions were extracted from these previous studies, classified and then mapped onto the WHO International Classification of Functioning, Disability and Health (WHO ICF) ${ }^{29}$ The results highlighted two domains and six key goal areas, which were incorporated into the GAS structure, along with their associated outcome parameters, to form the GAS-eous tool, as shown in table 2.

The first domain centres on 'symptoms and impairment' and includes spasticity-related pain relief, reduction of involuntary movements and improved/ maintained range of movement to prevent contractures and deformity. The second domain centres on 'activities and function' and includes passive function (making it 
Table 2 Classification of goals and measurement parameters that provide the framework for the GAS-eous tool

\begin{tabular}{|c|c|c|}
\hline Key goal areas & Goal definition & $\begin{array}{l}\text { Examples of recommended goal } \\
\text { parameters }\end{array}$ \\
\hline
\end{tabular}

\section{Domain 1. Symptoms/impairment}

Pain/discomfort (b280, b780)

Contracture prevention or

improvement (b710, b735)

Involuntary movements (b755, b760, b765)
Spasticity-related symptoms

- Pain (b280)

- Discomfort due to stiffness (b780)

Unwanted involuntary movements during use of other limbs

- Associated reactions (b755, b760)

- Spasms or dystonic movements (b765)

Improving range of movement (b735)

- Prevention of contractures/ deformity (b710)

- Splint application or tolerance

Ease of caring for the affected limb, for example:

- Maintaining hygiene of hand, elbow, axilla (b510)

- Maintaining skin integrity, cutting fingernails (d520)

- Dressing and positioning the limb, splint application

Active function

- Using the affected limb (d430, d440, d445)

Functional purpose (d500, d510, d540, d550, d560 d630, d640, d850, d920)

Mobility (d415, d420, d450)

\section{Other}

Cosmesis/body image

Therapy facilitation
Active motor tasks, for example:

- Reaching, grasp/release, holding/manipulating objects (d445)

- Fine dexterity (d440) and lifting/ carrying objects (d430)

Activities of daily living

- Personal: eating/drinking (d550, d560), self-care (d500, d510, d540)

- Extended activities of daily living (d630, d640)

- Occupational: work (d850) or recreation (d920)

- Ease of transfers (d420), balance, standing (d415)

- Walking (d450)
Improved mobility aesthetic appearance
Rating/10 (recorded in whole numbers using a verbal rating scale or a numbered graphic rating scale)

Carry angle of elbow/height of hand up torso during walking

Spasm frequency (number per day/night)

Percentage normal joint range (eg, $25 \%$, $50 \%, 75 \%)$

Ease of application (rating/10) or tolerance (hours per day)

Ability or time taken to complete defined task Rating of control or ease of movement/10

Ability or time taken to complete defined task

Rating on ArmA-active subscale

Time taken (eg, timed up and go)

Gait speed/endurance

Patient confidence, safety, frequency of trips/ falls

Patient's perception of body image, Patient satisfaction rating/10 Team's perception of interference Team rating/10 with therapy

Domains and key goal areas were identified in a multicentre analysis. ${ }^{7}$ The WHO International Classification of Functioning, Disability and Health codes are indicated. ${ }^{29}$ The recommended goal parameters may be used to define SMART goals within the selected areas. ArmA, Arm Activity measure; GAS-eous, Goal Attainment Scaling-Evaluation of Outcome for Upper Limb Spasticity; SMART, Specific, Measurable, Achievable, Realistic and Timed.

easier to care for the affected upper limb), active function (using the affected limb for everyday tasks or activities) and improving mobility (eg, maintaining balance, improved gait quality/efficiency). A small number of patients identified goals in other areas such as improving body image (cosmesis) and facilitating therapy. ${ }^{7}$

For the GAS-eous method, the patient and/or their family identify one primary and up to two secondary 
goals for treatment. Each goal is classified into one of the six key goal areas. SMART goal statements are then agreed on between the team and the patient/family, using one or more of the recommended goal parameters (wherever possible) to frame the SMART goal statement (see table 2).

The selected goal areas determine the standardised measures used alongside GAS within the ULS Index. Baseline and outcome GAS scores are recorded using the verbal rating scale in the GAS-light model. ${ }^{16}$ This translates into the five-point rating scale $(-2$ to +2$)$ and goal scores are combined using the GAS formula to derive an overall T-score using a standard formula. ${ }^{13}$

The GAS-eous method offers several potential advantages over traditional GAS. The more structured approach is designed to streamline goal setting in busy clinical settings, thus reducing the time taken to apply GAS. In addition, targeted selection of relevant standardised outcome measures reduces the time that would otherwise be spent recording numerous, less relevant measures. Importantly, GAS-eous retains the individualised nature of patient-centred goal setting focused on the patient's own priorities.

GAS is not just a measurement tool. Evidence suggests that engagement of patients in their own goal management can be an effective intervention in its own right. ${ }^{30}$ Alongside GAS-eous, ULIS-III will record patient engagement in goal setting and their satisfaction with the goals using six-point Likert scales designed specifically for this purpose. $^{21}$

Further information about the GAS-eous and associated tools may be found at the following websites: http://www.kcl.ac.uk/lsm/research/divisions/ cicelysaunders/resources/tools/gas-eous.aspx; http:// www.kcl.ac.uk/lsm/research/divisions/cicelysaunders/ resources/tools/gas.aspx.

\section{The ULSTR}

The ULSTR, a novel instrument designed for the ULIS programme, will record the physical interventions used to manage upper limb spasticity, and relate concomitant therapies to specific goal achievement. These interventions may include splinting, shoulder supports/slings, serial casting, upper limb positioning, stretches, neuromuscular electrical stimulation, strength training or task training. The ULSTR records both the type and intensity of interventions provided to patients. It was developed as part of the proof-of-principle study for ULIS-III as there was no tool available for relating patient goals to the concomitant therapies required for their achievement. The ULSTR is still undergoing full evaluation but will be used in ULIS-III as part of that process.

The ULSTR tool can be found at the following website: www.kcl.ac.uk/lsm/research/divisions/cicelysaunders/ resources/tools/Upper-Limb-focal-Spasticity-TherapyRecording-schedule-(ULSTR).aspx.

\section{The Spasticity-Related Quality-of-Life Tool}

SQoL-6D is another novel tool that was designed for the ULIS programme to further assess the quality of life benefits of BoNT-A treatment in patients with upper limb spasticity. It is a self-completed six-item questionnaire intended to evaluate quality of life in relation to upper limb spasticity within the key goal areas defined for GASeous (table 2).

SQoL-6D was developed because generic health utility measures, such as the Short Form-36 Health Survey and Assessment of Quality of Life instrument, have proven insensitive for evaluating change in quality of life with focal intervention for upper limb spasticity. ${ }^{15} \quad 31$ SQoL-6D is still undergoing development and evaluation in its original language (English) and has not yet been translated into other languages. ULIS-III will include piloting of the self-completed SQoL-6D in the subset of native English-speaking countries (Australia and the USA). It will be applied in this subgroup alongside the EuroQol Five Dimensions, Five Levels (EQ-5D-5L), a standardised generic health utility measure describing outcomes in five domains: mobility, self-care, usual activities, pain or discomfort, and anxiety/depression. ${ }^{32} 33$

The SQoL-6D tool can be found on the following website: www.kcl.ac.uk/lsm/research/divisions/ cicelysaunders/resources/tools/The-Spasticity-RelatedQuality-of-Life-Tool-(SQoL-6D).aspx.

\section{ULIS-III STUDY PROTOCOL: METHODS AND DESIGN}

The overall aims of ULIS-III are to describe real-life clinical practice and outcomes in the context of integrated upper limb spasticity management and to gain insights into which patients do best with which approaches to treatment over time. The trial protocol is available on ClinicalTrials.gov. ${ }^{34}$

\section{Study objectives}

The primary objective is to assess the attainment of patient-centred and function-related goals following repeated BoNT-A injections (where used) and alongside integrated upper limb spasticity management in real-life settings over a 2-year period. The primary outcome is the cumulated GAS T-score, defined as the mean of the individual GAS T-scores for all cycles per patient.

Secondary objectives are to:

- Describe the baseline characteristics of the study population, including demographics, duration and pattern of spasticity, and confounders (eg, severity of impairment).

- Describe injection practices and additional treatment strategies, and record clinical decision-making in the serial approach to spasticity management over time.

- Assess the attainment of patient-centred goals by goal area following each cycle of BoNT-A injections and overall attainment after repeated cycles, alongside standardised measures of symptoms, impairment, disability (activity limitation) and participation selected 
on an individual basis according to the goals for treatment.

- Describe and quantify concomitant therapy use and explore its relationship with goal attainment.

- Carry out a substudy of ULIS-III that will assess change in quality of life among a subpopulation of patients in Australia and the USA using generic and condition-specific instruments.

\section{Study design and setting}

ULIS-III is a 2-year, international, multicentre, observational, prospective, longitudinal cohort study that is expected to enrol over 1000 participants in 58 study centres across 14 countries. The study design is noninterventional and intended to reflect real-life clinical practice.

Study centres will be located in countries with marketing authorisation for at least one BoNT-A preparation for upper limb spasticity treatment. In addition, all eligible study centres must aim to follow the UK national consensus guidelines for the use of BoNT-A in the management of spasticity, ${ }^{1}$ and be able to collect data using GAS-eous and the tools included in the ULS Index.

The investigators' decision to prescribe BoNT-A must be taken prior to and independently from their decision to enrol the patient, as well as in accordance with routine clinical practice at the study site concerned.

\section{Ethics and dissemination}

ULIS-III is conducted in compliance with the Declaration of Helsinki, the Council for International Organizations of Medical Sciences International Ethical Guidelines for Epidemiological Studies ${ }^{35}$ and the guidelines for Good Pharmacoepidemiology Practices. ${ }^{36}$ Ethics approval will be sought at each site according to local legislation. All patients must provide written informed consent to participate in the study and to allow their medical data to be collected and analysed, or patient participation must be validated in accordance with local policy/guidelines.

An electronic data capture system (Viedoc 4, PCG Solutions AB, Uppsala, Sweden) will be used by the investigators to collect data in an electronic format; data will then be anonymised and transmitted to the sponsor for analysis.

Since ULIS-III is a non-interventional study, safety data will not be collected or analysed; however, standard regulations for reporting treatment-related spontaneous adverse events still apply within each country.

Study results will be presented at international meetings and published in peer-reviewed journals.

\section{Recruitment}

Patients are required to meet the following enrolment criteria:

- Adults, $\geq 18,20$ or 21 years of age dependent on local legislation, with upper limb spasticity, in whom a decision has already been made to inject BoNT-A.
- Patients may or may not have previously received BoNT-A injections.

- No previous participation in any other interventional clinical study of upper limb spasticity within the 12 weeks prior to their inclusion visit; no previous inclusion and subsequent withdrawal from this study.

A maximum of 20-30 patients per centre will be included. In order to avoid selection bias, patients in each centre will be recruited consecutively or according to a regular pattern.

\section{Treatment}

In order to replicate real-life settings, clinicians will make decisions regarding the type of BoNT-A preparation (Dysport (Ipsen Pharma, Paris, France), Botox (Allergen, Inc, Irvine, California, USA), Xeomin (Merz GMBH \& Co. KGaA, Frankfurt, Germany) or other) doses used, frequency of injection, target muscles for injection, number of injection points and volume injected per point on an individual patient basis. These decisions will be made according to usual clinical practice or in accordance with the current local summary of product characteristics.

Although recruitment to the study is triggered by a clinical decision to inject BoNT-A, thereafter patients will be followed up regardless of whether they have further injection cycles or not. Patients will undergo assessments and receive further BoNT-A injections at visits arranged according to the investigator's practice. End-of-assessment visits, or phone interviews for those participants who stopped treatment during the study, will be conducted no more than 24 months after the initial BoNT-A injection.

\section{Goal setting and goal attainment assessment}

Goal setting and goal attainment assessment will be implemented using the GAS-eous tool, ${ }^{518}$ as described above. Procedures to support a high standard of goal setting are detailed below.

Goal attainment will be recorded on a five-point numerical scale (range -2 to +2 ) and goal scores combined to give an aggregated T-score at each visit, using a standard formula. ${ }^{13}$ Overall goal attainment will be assessed using the cumulated GAS T-score.

\section{Outcome assessments}

The outcome measures for ULIS-III will primarily be captured using the ULS Index together with the ULSTR and the two health utility measures (the SQoL-6D and the EQ-5D-5L, native English-speaking subgroup only), as described above. Table 3 summarises how they will be applied throughout the study.

For the purposes of comparison with other studies, ULIS-III will also record the Disability Assessment Scale (DAS), ${ }^{37}$ defining the Principal Target for Treatment (PTT) at each visit.

As part of the ULIS Index, a global assessment of benefits will be performed by the investigator as well as the 
Table 3 Summary of outcome measures

\begin{tabular}{|c|c|}
\hline Outcome & Measure used \\
\hline \multicolumn{2}{|l|}{ Primary outcome } \\
\hline Patient-centred goal attainment & $\begin{array}{l}\text { Cumulated GAS T-score, defined per patient as the mean of the } \\
\text { individual GAS T-scores for all cycles through } 24 \text { months (in } \\
\text { predefined goal categories) derived using: } \\
\text { GAS-eous tool } \\
\text { Goal-related standardised measures within the ULS Index }\end{array}$ \\
\hline \multicolumn{2}{|l|}{ Secondary outcomes } \\
\hline - Muscle tone & $\rightarrow$ MAS \\
\hline $\begin{array}{l}\text { Severity of different aspects of impairment in both } \\
\text { upper limbs in patients with neurological damage }\end{array}$ & - ULS-NIS \\
\hline Treatment administration & - BoNT-A preparation INN \\
\hline Documentation of real-life injection practices & D Dose \\
\hline & - Date(s) of injection(s) \\
\hline & - Injection points and injection guidance (EMG, ES, US) \\
\hline & - Reasons for any changes to BoNT-A injection practice \\
\hline $\begin{array}{l}\text { Concomitant therapies (eg, specific interventions by } \\
\text { physiotherapists and occupational therapists) }\end{array}$ & ULSTR \\
\hline Patient-centred goal attainment over time by goal area & $\begin{array}{l}\text { Cumulated GAS T-score of all goals assessed within each goal } \\
\text { area }\end{array}$ \\
\hline $\begin{array}{l}\text { Attainment of treatment goals after each cycle of BoNT-A } \\
\text { injections }\end{array}$ & Changes in the GAS T-score for each injection cycle \\
\hline $\begin{array}{l}\text { Percentage achievement of treatment goals per goal } \\
\text { area }\end{array}$ & Documented overall and per injection cycle \\
\hline Evolution of goal changes across the study & $\begin{array}{l}\text { Recorded alongside changes from baseline in the standardised } \\
\text { outcome measures }\end{array}$ \\
\hline $\begin{array}{l}\text { Correlations of patient-centred goals and related } \\
\text { standardised rating scales }\end{array}$ & $\begin{array}{l}\text { GAS-eous and standardised outcome measures selected } \\
\text { according to the goals for treatment within the ULS Index (NGRS } \\
\text { or SPIN, ArmA, FAC, ARRS) }\end{array}$ \\
\hline Change in disability & $\begin{array}{l}\text { Change from baseline in the four domains of DAS (hygiene, } \\
\text { dressing, limb position and pain) } \\
\text { PTT will be defined at each visit, based on one of the four DAS } \\
\text { domains }\end{array}$ \\
\hline Global assessment of benefits & By investigator and caregiver/patient \\
\hline Change in quality of life* & $\begin{array}{l}\text { EQ-5D-5L } \\
\text { SQoL-6D }\end{array}$ \\
\hline
\end{tabular}

*Only in subpopulations of patients in the USA and Australia.

ArmA, Arm Activity measure; ARRS, Associated Reaction Rating Scale; BoNT-A, botulinum toxin A; DAS, Disability Assessment Scale; EMG, electromyography; EQ-5D-5L, EuroQol Five Dimensions, Five Levels; ES, electrostimulation; FAC, Functional Ambulation Category; GAS, goal attainment scaling; GAS-eous, Goal Attainment Scaling-Evaluation of Outcomes for Upper Limb Spasticity; INN, international non-proprietary name; MAS, Modified Ashworth Scale; NGRS, Numbered Graphic Rating Scale; PTT, principal target of treatment; SPIN, Scale of Pain Intensity; SQoL-6D, Spasticity-Related Quality-of-Life Tool; ULS Index, Upper Limb Spasticity Index; ULS-NIS, Upper Limb Spasticity-adapted version of the Neurological Impairment Scale; ULSTR, Upper Limb Focal Spasticity Therapy Recording Schedule; US, ultrasound.

patient and/or caregiver, with changes from previous visits classified as: much worse $(-2)$, worse $(-1)$, no change $(0)$, some benefit $(+1)$ or great benefit $(+2)$.

\section{Health economic evaluation}

Healthcare resource utilisation, including direct clinical costs, indirect costs associated with spasticity and concomitant medications, will be assessed at baseline and at every subsequent visit. These will include assessment of changes in concomitant treatments, such as medications associated with spasticity and pain, changes in employment status and maintenance of healthcare, for example, nurse or caregiver. ULSTR data will be used to determine healthcare resource use associated with upper limb spasticity-related physical therapy.

Utility data (quality-adjusted life-years (QALYs)) for the subgroups of patients in native English-speaking countries will be derived using data from the EQ-5D-5L and SQoL-6D assessment tools.

\section{Study schedule and assessments}

The nature and timing of patient assessments will be in accordance with routine clinical practice at the individual study centres. It is expected that some patients will 
discontinue follow-up within the 2-year period, as spasticity can be a self-limiting condition. End-of-study visits or telephone interviews will be conducted 24 months after recruitment to ascertain the patient's condition and reasons for discontinuing follow-up, if this was the case.

All primary and secondary goals and outcome assessment data will be documented in eCRFs at each visit. To ensure data consistency and accuracy, all amendments to eCRF data once entered will be automatically tracked and a reason for change will be required.

\section{Planned statistical analysis}

All statistical evaluations will be performed using the Statistical Analysis System (SAS V.9 or later; SAS Institute, Inc, Cary, North Carolina, USA) according to a preplanned analysis strategy. No formal treatment comparisons will be made; however, results will be reported descriptively with $95 \%$ CIs and $\mathrm{p}$ values presented for exploratory purposes only. A $p$ value of $<0.05$ will be considered to indicate statistical significance.

\section{Sample size calculation}

Sample size was calculated on the basis of detecting an effect size of 0.5 in the primary end point of cumulated GAS T-score across the full study. Assuming a type I error of $5 \%$ (two-sided) and a power of $\geq 90 \%, \geq 800$ patients will allow comparisons between seven goal areas based on cumulated GAS T-scores. This will also allow comparisons with $\geq 80 \%$ power in the case of an unbalanced distribution of up to $3: 1$ between goal areas. Assuming a $20 \%$ dropout rate, the target recruitment number is $\geq 1000$ patients.

\section{Effectiveness evaluation}

The cumulated GAS T-score, defined as the mean of the GAS T-scores for each individual patient for all visits over the 24-month study period, will be presented descriptively using summary statistics.

Cumulated GAS T-scores, calculated by goal area, will be compared descriptively, using an analysis of variance model and individual pairwise comparisons, to assess the relative attainment of goals between the different goal areas.

The correlation between goal attainment and change from baseline (visit 1) in the associated standardised measures will be assessed using Spearman's rank correlation coefficients. The observed correlation coefficients will be assessed for difference from zero using a permutation test and $\mathrm{p}$ values will be presented. All other secondary end points will be reported descriptively.

\section{Exploratory analyses}

The relationship between primary outcome (cumulated GAS T-score) and intervention programme (BoNT-A and physical therapy interventions) will be explored descriptively. A stepwise linear regression model will be used to identify prognostic factors for response, which is defined as an improvement from baseline GAS T-score of at least +10 points. In addition, descriptive analyses will be carried out to evaluate and describe any possible country and centre effects.

\section{Health economics analysis}

Estimated direct and indirect healthcare costs associated with upper limb spasticity will be presented descriptively based on assigning unit costs derived from established sources of unit cost data to the healthcare resources used. The sources of unit cost data will be determined at a data review meeting. Cost-effectiveness ratios for cost per response will be based on patients' Disability Assessment Score achievement, which is defined as an improvement of $\geq 1$ point in the DAS score in the principal target of treatment at the end of the study compared with visit 1 , with separate analyses performed for each domain. GAS outcomes will also be evaluated for these subgroups. In addition, analyses will be performed on the US and Australian subgroups to evaluate cost per QALY and cost-utility.

\section{Trial status}

Recruitment began in January 2015 and patient participation duration is 2 years. It is estimated that the enrolment target of 1000 patients will be achieved by December 2016 and the final analysis of patient data is expected to be completed in the first quarter of 2019 .

\section{QUALITY EVALUATION OF GOAL SETTING}

The validity of GAS depends on a high standard of goal setting and documentation of SMART goal statements. Goals should be centred on improvement at the level of function, as opposed to impairment, as defined by the WHO ICF. ${ }^{29}$

In ULIS-II, an interim validation process was undertaken part-way through recruitment to ensure that clinicians were setting SMART function-related goals in accordance with the training. ${ }^{18}$ Goal statements were examined on a centre-by-centre basis and investigators were blinded to country and centre. Statements were assessed on two criteria: the WHO ICF domain for function (A, B, C); and the quality of the SMART description $(-$ to ++$)$, as previously described. ${ }^{18}$ It was accepted that, for some patients, goal statements would be impairment-related (eg, prevention of contractures). However, investigators expected that at least some goals from each centre would be related to function. The clinicians received feedback and had the opportunity to improve the quality of their goal statements for patients who had not yet returned for their second visit. This led to documented improvement, but by the end of the study only $25 \%$ of centres achieved the top rating $(\mathrm{A}++) .{ }^{18}$

Part of the rationale for development of the GAS-eous tool was to improve the quality of goal setting, and the recruiting centres (many of which also recruited for ULIS-II) have undergone further training for ULIS-III. Goal reviews, validation and feedback by the Steering 
Group will also be conducted at more frequent intervals throughout the recruitment phase to facilitate a high standard of goal setting.

Participant recruitment was initiated in January 2015. By the first goal review in June 2015, 58 sites had been selected globally, over half had received training on the study and 15 sites had started recruitment across five countries, including Australia, Austria, France, Russia and Poland. Initial patient data have been collected for 79 patients across 13 sites in three countries. Of these 79 patients, 42 set primary goals only, 24 also set one secondary goal and 13 set two secondary goals in addition to a primary goal. Goal setting data were quality checked and centres were given a rating based on the WHO ICF and SMART guidelines, as described above. ${ }^{18}$ As shown in table $4,11 / 13$ centres achieved the highest rating (A++).

Goals were organised into two domains and subdivided into six key goal areas. The most common areas for primary goals were passive function $(30 / 79(38.0 \%))$ followed by active function $(17 / 79(21.5 \%))$ and pain $(17 / 79(17.7 \%))$. Although the percentages varied slightly, the total 129 goals, including secondary goals, represented a broadly similar pattern. Only two primary goals did not fit into these six key goal areas-these addressed improvement of cosmesis (perception of body image) and the facilitation of therapy. These findings provide support for the validity of the goals classification that underpins the GAS-eous tool.

\section{DISCUSSION}

The ULIS programme sets in train a methodology that is practical for use in routine clinical practice and in

\begin{tabular}{lccl} 
Table 4 & Goals set and centre ratings at visit 1 \\
\hline $\begin{array}{l}\text { Country/ } \\
\text { centre ID }\end{array}$ & $\begin{array}{l}\text { Number of } \\
\text { primary } \\
\text { goals set }\end{array}$ & $\begin{array}{l}\text { Number of } \\
\text { secondary } \\
\text { goals set }\end{array}$ & $\begin{array}{l}\text { Centre } \\
\text { rating }\end{array}$ \\
\hline $1 / 4$ & 1 & 0 & Not entered \\
& & & at time of \\
& & & data \\
& & 2 & extraction \\
$1 / 5$ & 1 & 5 & $\mathrm{~A}++$ \\
$1 / 6$ & 5 & 0 & $\mathrm{~A}++$ \\
$1 / 8$ & 1 & 0 & $\mathrm{~A}++$ \\
$2 / 1$ & 4 & 5 & $\mathrm{~A}-$ \\
$2 / 2$ & 15 & 2 & $\mathrm{~A}++$ \\
$2 / 4$ & 24 & 8 & $\mathrm{~A}++$ \\
$3 / 1$ & 4 & 15 & $\mathrm{~A}++$ \\
$3 / 2$ & 12 & 2 & $\mathrm{~A}++$ \\
$3 / 3$ & 2 & 3 & $\mathrm{~A}++$ \\
$3 / 5$ & 2 & 3 & $\mathrm{~A}++$ \\
$3 / 6$ & 2 & 5 & $\mathrm{~A}++$ \\
$3 / 7$ & 6 & &
\end{tabular}

Rating: A, Some goal statements contain reference to functional activities at the level of disability or participation; ++, there is a specific, measurable, achievable, realistic and timed goal description, sufficiently detailed and specific to make an accurate goal attainment scaling rating; -, no clear goal description. future studies. This methodology will enable studies to expand their clinical data set to one of sufficient size to elucidate important findings, including which patients are most likely to benefit from what types of treatment and how best to capture the outcomes.

ULIS-III will build on previous studies that have used goal attainment to evaluate outcomes from upper limb spasticity interventions. ${ }^{5638}$ It will expand the patient cohort and capture the benefits of treatment in real-life clinical practice over several treatment cycles. ULIS-III will use a more structured approach to outcome measurement incorporating both GAS and standardised measures in a targeted way that is feasible to implement in clinical practice. In addition, it will assess the wider benefits of BoNT-A treatment for upper limb spasticity by evaluating economic and quality of life data, as well as concomitant therapies.

Initial data from the inclusion visits of 79 patients across 13 centres in three countries provide support for the validity of the goals classification that underpins the GAS-eous tool, and demonstrate a high standard of goal setting in the majority of centres.

\section{Strengths and limitations}

The strengths of the ULIS-III approach include the wide international representation of participating centres, which will capture the experience of clinicians from around the globe, ensuring the generalisability of results. The use of patient-centred goal setting and GAS in ULIS-III will enable the assessment of outcomes that are important to the patient and/or their family while maintaining realistic expectations for the outcome. In addition, the structured approach to GAS in parallel with standardised measurement may improve the comparability of results across different settings. Moreover, a high standard of goal setting is supported by a comprehensive GAS training programme and interim goal reviews.

However, there are recognised limitations of the ULIS-III approach. Recruitment is restricted to 20-30 patients per centre, which may introduce some selection bias through under-representation of less common presentations of spasticity. In addition, the use of tools that are still undergoing evaluation (ULSTR and SQoL-6D) means that the data generated from these will be less robust. However, they may help to fill gaps in knowledge for which no prevalidated tools exist currently.

Despite the recognised limitations, ULIS-III will provide a unique and important set of information regarding treatment with and outcomes from BoNT-A in real-life management of upper limb spasticity worldwide. The present methodology will help to ensure credibility of results from ULIS-III, and will also underpin future studies and inform other clinical trials and cohort studies in this context.

The ULIS Programme Steering Group

- Expert clinicians: LT-S (clinical lead), SA, KF, JJ

- Ipsen staff: JB, PM, Hugues Berard 
Author affiliations

${ }^{1}$ Department of Palliative Care, Policy and Rehabilitation and Regional Rehabilitation Unit, King's College London School of Medicine, Northwick Park Hospital, London, UK

${ }^{2}$ Centro de Medicina de Reabilitaçãode Alcoitão, Serviço de Reabilitação de Adultos, Estoril, Portugal

${ }^{3}$ Medical Affairs, Ipsen Pharma, Boulogne-Billancourt, France

${ }^{4}$ Department of Neurorehabilitation, Gailtal-Klinik, Hermagor, Austria

Acknowledgements This work was supported by Ipsen Pharma. The authors thank all the investigators and patients who have participated in the ULIS programme and, in particular, Steve De Graaff and lan Baguley, Australia. They also acknowledge the work of the ULIS-III project managers at Ipsen, Isabelle Gueguen and Elodie Blouquit. In addition, the authors would like to acknowledge the local coordinating investigators involved in training local investigators: Ian Baguley, Mario Nunzio Manca, Tae Mo Chung, Andrzej Friedman, Alexis Schnitzler, Jorge Hernandez Franco, Svetlana Khatkova and Raymond Rosales. They would also like to acknowledge the editorial assistance of Watermeadow Medical. Ipsen Pharma provided financial support for this assistance. Financial support for manuscript preparation was also provided through the Dunhill Medical Trust.

Contributors The protocol for ULIS-III was developed by Ipsen Pharma in conjunction with the ULIS Programme Steering Group. LT-S wrote the first draft of this manuscript but all authors were involved in its critique, adjustment and final approval. LT-S, SA, KF and JJ were involved in data collection and assembly of data. PM and JB were involved in the concept and design, and the plan for data analysis was led by PM.

Funding Ipsen Pharma.

Competing interests LT-S, SA, KF and JJ all received honoraria and conference attendance fees from Ipsen for undertaking this research. LT-S and SA have a specific interest in outcomes evaluation and have published extensively on the use of GAS in this context, as well as a number of the other standardised measures (including the ARRS, the ArmA and the Neurological Impairment Scale). All of these tools are freely available, however, and they have no personal financial interest in any of the material mentioned in this article. SA receives funding from the National Institute of Health Research (NIHR) in the form of a Clinical Lectureship award and an NIHR CLAHRC Northwest London fellowship. KF has a specific interest in outcomes evaluation and the use of the International Classification of Function in clinical settings. JJ has a particular interest in clinical and instrumental spasticity evaluation methods, goal setting, treatment strategies/techniques and outcome measurement. PM and JB are employees of Ipsen.

Disclaimer The views expressed are those of the authors and not necessarily those of the National Health Service, UK (NHS), the NIHR, NIHR CLAHRC Northwest London or the Department for Health, UK.

Ethics approval See Health Authorities list here: https://clinicaltrials.gov/ct2/ show/study/NCT02454803.

Provenance and peer review Not commissioned; externally peer reviewed.

Open Access This is an Open Access article distributed in accordance with the Creative Commons Attribution Non Commercial (CC BY-NC 4.0) license, which permits others to distribute, remix, adapt, build upon this work noncommercially, and license their derivative works on different terms, provided the original work is properly cited and the use is non-commercial. See: http:// creativecommons.org/licenses/by-nc/4.0/

\section{REFERENCES}

1. Royal College of Physicians, British Society of Rehabilitation Medicine, Chartered Society of Physiotherapy, Association of Chartered Physiotherapists Interested in Neurology. Spasticity in adults: management using botulinum toxin. National guidelines. London, UK: RCP, 2009. https://www.rcplondon.ac.uk/sites/default/ files/documents/spasticity-in-adults-management-botulinum-toxin.pdf (accessed Feb 2015).

2. Sheean G. The pathophysiology of spasticity. Eur J Neurol 2002;9 (Suppl 1):3-9.
3. Brown P. Pathophysiology of spasticity. J Neurol Neurosurg Psychiatr 1994;57:773-7.

4. Gracies JM, Brashear A, Jech R, et al. Safety and efficacy of abobotulinumtoxinA for hemiparesis in adults with upper limb spasticity after stroke or traumatic brain injury: a double-blind randomised controlled trial. Lancet Neurol 2015;14:992-1001.

5. Turner-Stokes L, Fheodoroff K, Jacinto J, et al. Results from the Upper Limb International Spasticity Study-II (ULIS-II): a large, international, prospective cohort study investigating practice and goal attainment following treatment with botulinum toxin $\mathrm{A}$ in real-life clinical management. BMJ Open 2013;3:e002771.

6. Turner-Stokes L, Baguley I, De Graff S, et al. Goal attainment scaling in the evaluation of treatment of upper limb spasticity with botulinum toxin: a secondary analysis from a double blind placebo controlled randomised clinical trial. J Rehabil Med 2010;42:81-9.

7. Ashford S, Fheodoroff K, Jacinto J, et al. Common goal areas in the treatment of upper limb spasticity: a multicentre analysis. Clin Rehabil 2016;30:617-22.

8. Scottish Intercollegiate Guidelines Network. Management of patients with stroke: rehabilitation, prevention and management of complications, and discharge planning. A National Clinical Guideline. Edinburgh, Scotland: Scottish Intercollegiate Guidelines Network, 2010. http://www.sign.ac.uk/pdf/sign118.pdf (accessed Dec 2014).

9. Holliday RC, Cano S, Freeman JA, et al. Should patients participate in clinical decision making? An optimised balance block design controlled study of goal setting in a rehabilitation unit. J Neurol Neurosurg Psychiatry 2007;78:576-80.

10. Brown M, Levack W, McPherson KM, et al. Survival, momentum, and things that make me "me": patients' perceptions of goal setting after stroke. Disabil Rehabil 2014;36:1020-6.

11. Bakheit AM, Zakine $B$, Maisonobe $P$, et al. The profile of patients and current practice of treatment of upper limb muscle spasticity with botulinum toxin type A: an international survey. Int $J$ Rehabil Res 2010;33:199-204.

12. Levack WM, Dean SG, Siegert RJ, et al. Navigating patient-centered goal setting in inpatient stroke rehabilitation: how clinicians control the process to meet perceived professional responsibilities. Patient Educ Couns 2011;85:206-13.

13. Kiresuk T, Sherman R. Goal attainment scaling: a general method of evaluating comprehensive mental health programmes. Community Ment Health J 1968;4:443-53.

14. Ashford S, Turner-Stokes L. Goal attainment for spasticity management using botulinum toxin. Physiother Res Int 2006;11:24-34.

15. McCrory $\mathrm{P}$, Turner-Stokes L, Baguley IJ, et al. Botulinum toxin A for treatment of upper limb spasticity following stroke: a multi-centre randomized placebo-controlled study of the effects on quality of life and other person-centred outcomes. J Rehabil Med 2009;41:536-44.

16. Turner-Stokes L. Goal attainment scaling (GAS) in rehabilitation: a practical guide. Clin Rehabil 2009;23:362-70.

17. Tennant A. Goal attainment scaling: current methodological challenges. Disabil Rehabil 2007;29:1583-8.

18. Turner-Stokes L, Fheodoroff K, Jacinto J, et al. Upper limb international spasticity study: rationale and protocol for a large, international, multicentre prospective cohort study investigating management and goal attainment following treatment with botulinum toxin A in real-life clinical practice. BMJ Open 2013;3:e002230.

19. Bohannon RW, Smith MB. Interrater reliability of a modified Ashworth scale of muscle spasticity. Phys Ther 1987;67: 206-7.

20. Turner-Stokes L, Thu A, Williams $\mathrm{H}$, et al. The Neurologica Impairment Scale: reliability and validity as a predictor of functional outcome in neurorehabilitation. Disabil Rehabil 2014;36:23-31.

21. Turner-Stokes L, Rose $H$, Ashford $S$, et al. Patient engagement and satisfaction with goal planning: impact on outcome from rehabilitation. Int J Ther Rehabil 2015;22:210-16.

22. Jackson D, Horn S, Kersten $\mathrm{P}$, et al. Development of a pictorial scale of pain intensity for patients with communication impairments: initial validation in a general population. Clin Med 2006;6:580-5.

23. Macfarlane A, Turner-Stokes L, De Souza L. The associated reaction rating scale: a clinical tool to measure associated reactions in the hemiplegic upper limb. Clin Rehabil 2002;16:726-35.

24. Ashford S, Turner-Stokes L, Siegert RJ, et al. Initial psychometric evaluation of the Arm Activity measure (ArmA): a measure of active and passive function in the hemiparetic arm. Clin Rehabil 2013;27:728-40.

25. Holden MK, Gill KM, Magliozzi MR, et al. Clinical gait assessment in the neurologically impaired. Reliability and meaningfulness. Phys Ther 1984;64:35-40. 
26. Turner-Stokes L, Ashford S, De Graaff S, et al. The GAS-EOUS tool -a framework for evaluation of outcome in upper limb spasticity. Neurorehabil Neural Repair 2012;26:695-804.

27. Ashford S, Turner-Stokes L. Management of shoulder and proximal upper limb spasticity using botulinum toxin and concurrent therapy interventions: a preliminary analysis of goals and outcomes. Disabil Rehabil 2009;31:220-6.

28. Turner-Stokes L, Williams $\mathrm{H}$, Ashford S. A UK pilot for a large international prospective cohort analysis of the use of botulinum toxin A (BoNT-A) in upper limb spasticity. PM R 2012;2:S190-91.

29. WHO. International Classification of Functioning, Disability and Health. Geneva, Switzerland: World Health Organization, 2002.

30. McPherson KM, Kayes N, Weatherall M. A pilot study of self-regulation informed goal setting in people with traumatic brain injury. Clin Rehabil 2009;23:296-309.

31. Childers MK, Brashear A, Jozefczyk P, et al. Dose-dependent response to intra-muscular botulinum toxin type $A$ for upper limb spasticity after a stroke. Arch Phys Med Rehabil 2004;85:1063-9.

32. Herdman M, Gudex C, Lloyd A, et al. Development and preliminary testing of the new five-level version of EQ-5D (EQ-5D-5L). Qual Life Res 2011;20:1727-36.
33. Oemar M, Janssen B. EQ-5D-5L User Guide: Basic Information on How to Use the EQ-5D-5L Instrument. Version 2.0. October 2013. http://www.euroqol.org (accessed Feb 2015).

34. Ipsen. Attainment of Person-centred Goals After Botulinum Toxin Treatment for Upper Limb Spasticity in Real Life Practice (ULIS III). https://clinicaltrials.gov/ct2/show/study/NCT02454803? term=ULIS+III+upper+limb+spasticity\&rank=1 (accessed Nov 2015).

35. Council for International Organizations of Medical Sciences (CIOMS). International eithical guidelines on epidemiological studies. Geneva: CIOMS, 2009.

36. International Society for Pharmacoepidemiology. Guidelines for Good Pharmacoepidemiology Practices (GPP). 2007. http://www. pharmacoepi.org/resources/guidelines_08027.cfm (accessed Nov 2015).

37. Brashear A, Zafonte R, Corcoran M, et al. Inter- and intrarater reliability of the Ashworth Scale and the Disability Assessment Scale in patients with upper upperlimb poststroke spasticity. Arch Phys Med Rehabil 2002;83:1349-54.

38. Ward $A B$, Wissel J, Borg J, et al. Functional goal achievement in post-stroke spasticity patients: the BOTOX® Economic Spasticity Trial (BEST). J Rehabil Med 2014;46:504-13. 